\title{
Pathological severity determines the renal recovery for anti-myeloperoxidase antibody-associated vasculitis requiring dialysis at disease onset: a retrospective study
}

Peng-cheng $\mathrm{Xu}^{{ }^{*}}{ }^{*}$, Tong Chen ${ }^{2}$, Si-jing Wu${ }^{1}$, Xia Yang ${ }^{1}$, Shan Gao ${ }^{1}$, Shui-yi Hu${ }^{1}$, Li Wei ${ }^{1}$ and Tie-kun Yan ${ }^{1}$

\begin{abstract}
Background: Many patients with anti-neutrophil cytoplasmic antibody (ANCA)-associated vasculitis (AAV) need dialysis at disease onset due to severe kidney injury. Determining whether they can become dialysis independent is an important clinical assessment.

Methods: Forty kidney biopsy-proved myeloperoxidase (MPO)-ANCA associated AAV patients who required dialysis at disease onset were enrolled. Relationships between laboratory and pathological characteristics and prognoses were analyzed.

Results: Twenty-five patients obtained dialysis independence within 3 months, while the other 15 patients remained dialysis dependent. No sclerotic class was identified among the 40 patients. Only two biopsies exhibited focal class diagnoses and both these patients recovered their renal function. The renal recovery rate of the 20 patients with mixed class was significantly lower than that of the 18 patients with crescentic class (40.0\% vs. 83.3\%, $p=0.006)$. Receiver operating characteristics (ROC) curves showed fibrous crescent+global glomerulosclerosis greater than $32.6 \%$ was a strong predictor of dialysis dependence with a sensitivity of $93.3 \%$ and specificity of $88.0 \%$. When the percentage of fibrous crescent+global glomerulosclerosis exceeded $47.9 \%$, dialysis independence was not possible. Correlation analysis indicated that platelet counts were negatively correlated with the percentage of fibrous crescent+global glomerulosclerosis $(R=-0.448, p=0.004)$. Most patients with increased platelets $(84.62 \%)$ obtained renal recovery. Compared with methylprednisolone pulse therapy, plasma exchange accelerated renal recovery (29.4 \pm 15.6 vs. $41.4 \pm 11.7$ days, $p=0.039)$.

Conclusions: For MPO-ANCA AAV who required dialysis at disease onset, crescentic and mixed classes accounted for the majority of patients in our cohort. The renal outcome of mixed class patients was worse than that of crescentic class. A high proportion of fibrous crescent+global glomerulosclerosis is a predictor of dialysis dependence. Increased platelet count is associated with active and reversible renal lesions.
\end{abstract}

Keywords: Antineutrophil cytoplasmic antibody, Myeloperoxidase, Renal biopsy, Histopathologic classification, Dialysis

\footnotetext{
*Correspondence: nkxpc@163.com

'Department of Nephrology, Tianjin Medical University General Hospital,

Tianjin 300052, China

Full list of author information is available at the end of the article
}

(c) The Author(s). 2019 Open Access This article is distributed under the terms of the Creative Commons Attribution 4.0 International License (http://creativecommons.org/licenses/by/4.0/), which permits unrestricted use, distribution, and reproduction in any medium, provided you give appropriate credit to the original author(s) and the source, provide a link to the Creative Commons license, and indicate if changes were made. The Creative Commons Public Domain Dedication waiver (http://creativecommons.org/publicdomain/zero/1.0/) applies to the data made available in this article, unless otherwise stated. 


\section{Background}

Anti-neutrophil cytoplasmic antibody (ANCA)-associated vasculitis (AAV) is a systemic vasculitis. The kidney injury manifests as a rapidly progressive glomerulonephritis (RPGN), and this is very common in AAV and affects disease prognosis [1, 2]. Kidney biopsy typically reveals pauci-immune necrotizing glomerulonephritis (GN) $[3,4]$. In AAV, a percentage of patients require renal replacement therapy at disease onset. Some of these patients enter end stage renal disease (ESRD) rapidly in spite of intensive immunosuppressive therapy [5].

Without treatment, the prognosis of AAV is very poor. Immunosuppressive therapy effectively increases survival. However, since intensive immunosuppressive therapy can lead to fatal infection [6], reducing the intensity of immunosuppressive therapy is necessary for patients who will not recover renal function. Based on the results of previous studies, plasma exchange (PE) could increase the rate of renal function recovery in AAV [7], but not all patients recover renal function with PE. Determining whether patients with severe kidney injury have the potential to become dialysis independent is of great importance in clinical practice.

According to the pathological classification proposed by Berden $\mathrm{AE}$ et al. [8], the pathological manifestations of ANCA-GN can be classified as focal, crescentic, mixed and sclerotic categories. This classification has been demonstrated to be effective in predicting the risk of ESRD, but there are no studies on whether the pathological characteristics can be used to assess the possibility of renal function recovery for patients who require dialysis at disease onset. To investigate this, 40 kidney biopsy-diagnosed ANCA-GN patients who required dialysis at disease onset were enrolled in our study.

\section{Materials and methods}

\section{Participants}

Between January 2005 and May 2017, 244 patients with AAV were diagnosed at the Tianjin Medical University General Hospital. All patients fulfilled the Chapel Hill Consensus Conference classification [9]. Only 7 patients were proteinase 3 (PR3)-ANCA positive, all other patients were myeloperoxidase (MPO)-ANCA positive. Patients with PR3-ANCA were excluded because of insufficient numbers. Among the 237 patients with MPO-ANCA, 217 patients had kidney injury (including haematuria, proteinuria and abnormality of kidney function), but 102 patients did not undergo kidney biopsy. Among these 102 patients, 74 patients did not agree to kidney biopsy (Sixteen patients needed dialysis at presentation and 10 patients recovered kidney function within 3 months after treatment) and 28 patients did not receive kidney biopsy because of the insufficient thickness of kidney cortex (Twelve patients needed dialysis at presentation and none recovered kidney function within 3 months following treatment). Among the 115 patients who received kidney biopsy, 46 patients required dialysis at disease onset. In the current study, dialysis dependence was defined as when the patients could not acquire dialysis independence for at least 3 months after treatment, so 6 patients were excluded because they died before getting dialysis independence within 3 months. In summary, 40 patients were included in this study (Fig. 1). The research was in compliance of the declaration of Helsinki and the protocol was approved by the institutional review board of Tianjin Medical University General Hospital. Informed consent was not required because the study was retrospective.

\section{Clinical and laboratory findings}

Clinical data included the following: gender, age (years), time from first symptom (days) and the level of Birmingham Vasculitis Activity Score (BVAS). Blood samples were taken for laboratory test. Estimated glomerular filtration rate (eGFR) was calculated with an equation described previously [10].

\section{Renal histopathology}

Renal biopsies were performed and evaluated using direct immunofluorescence and light microscopy by two pathologists. According to the pathological classification described previously, the biopsy was classified as focal, crescentic, mixed and sclerotic class [8]. The presence of fibrinoid necrosis, cellular crescents and global glomerulosclerosis of each glomerulus was scored. The presence of glomerular lesions was calculated as the percentage of the total number of glomeruli. Interstitial fibrosis $(-/+/++)$, tubular atrophy $(-/+/++)$ and arterial wall thickening $(-/+)$ were scored semiquantitatively [11].

\section{Treatment}

All patients received hemodialysis following admission. Indications of dialysis included serum creatinine $>500 \mu \mathrm{mol} / \mathrm{L}$, severe electrolyte and acid-base abnormalities, volume overload, pericarditis and encephalopathy [12]. Dialysis was terminated when eGFR surpassed $15 \mathrm{ml} / \mathrm{min} / 1.73 \mathrm{~m}^{2}$ and there was no hyperkalemia, heart failure or edema. In the induction of remission, all patients received corticosteroid treatment. Oral or intravenous corticosteroid was prescribed at an initial dosage of $1 \mathrm{mg} / \mathrm{kg} /$ day for 1 month, with reducing doses over time. Due to the severe kidney injury, it was recommended that all patients receive PE treatment, but due to restrictions in the plasma supply, only 14 patients received PE treatment $(2000 \mathrm{ml}$ per treatment session, 3-10 treatment sessions). The other 26 patients who could not be provided enough plasma did not receive PE treatment; they received corticosteroid pulse therapy. Intravenous methylprednisolone pulse therapy (MP) was prescribed for 3 


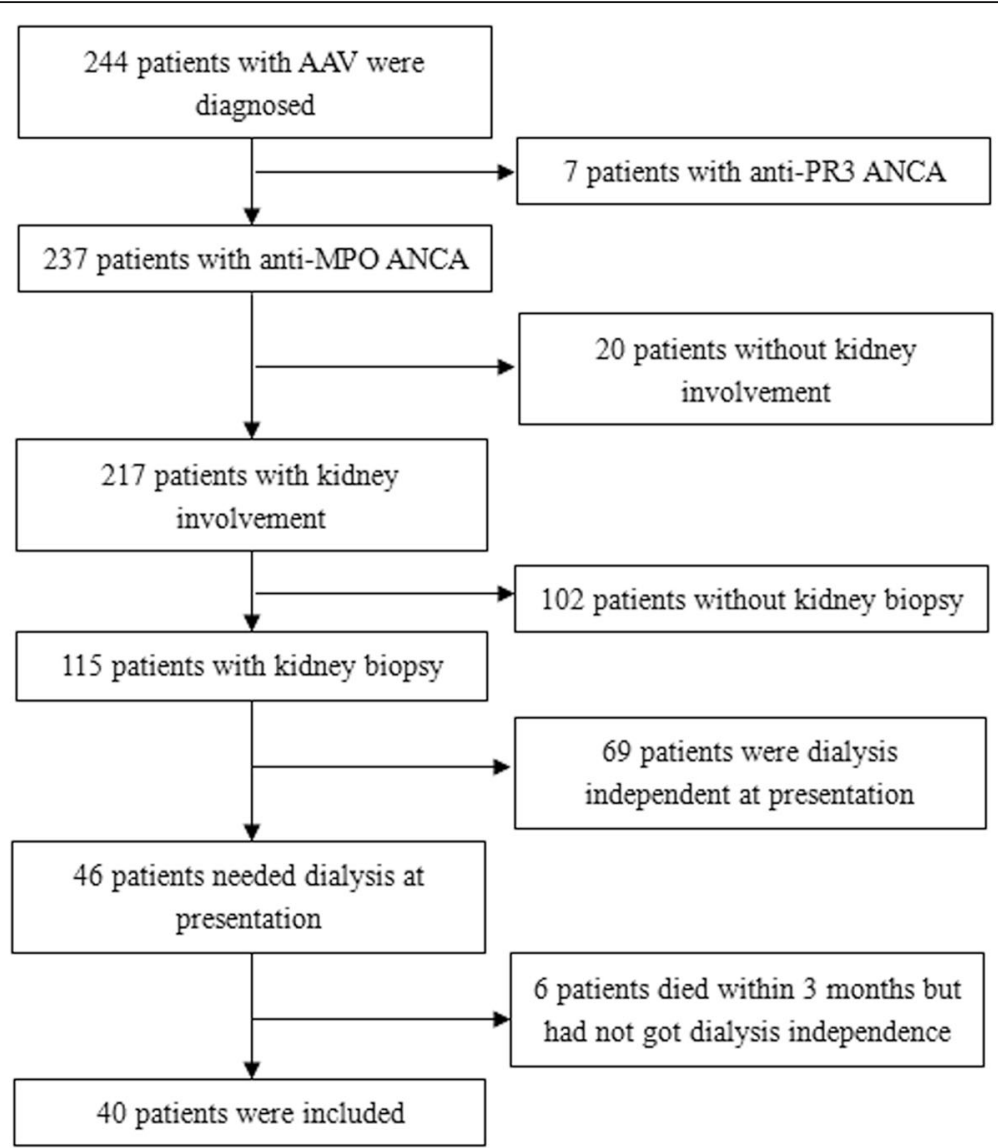

Fig. 1 Patients selection flowchart. A total of 244 patients with AAV were diagnosed. Only 7 patients were PR3-ANCA positive and were excluded. Among 237 patients with MPO-ANCA, 217 patients had kidney injury, but 102 patients did not receive kidney biopsy. Patients without kidney biopsy and patients who died before obtaining dialysis independence within 3 months were also excluded. At last, 40 patients were included in this study

consecutive days (200-500 mg/d) and the MP regimen was repeated after a week if necessary. The majority of patients (31 of 40) received intravenous or oral cyclophosphamide (CTX). $400 \mathrm{mg}$ CTX was administered intravenously every two weeks (Pulse CTX) or by daily oral dose of $50-100 \mathrm{mg}$ according to body weight (Oral CTX). Oral corticosteroid+CTX or corticosteroid+mycophenolate $(0.5-2 \mathrm{~g} / \mathrm{d})$ was administered for more than 1 year for maintenance therapy. Oral prednisone was prescribed at an initial dosage of $1 \mathrm{mg} / \mathrm{kg} /$ day for $1 \mathrm{month}$, with reducing doses over time. No patient received daily oral azathioprine (AZA) treatment because AZA was not available in our hospital.

\section{Statistical analyses}

Differences of quantitative parameters were assessed using one-way ANOVA tests (for data that were normally distributed) or nonparametric test (for data that were not normally distributed). Categorical variables are presented as frequencies. Survival was calculated with the Kaplan-Meier, and the curves were compared by
Log-Rank test. To calculate the area under the curve (AUC) value, receiver operating characteristics (ROC) curves were generated. The cut-off value was confirmed by calculating Youden index. For correlation analysis the spearman test was used. $P$ values lower than 0.05 were considered significant. The software SPSS, version 19.0 for Windows (IBM, Chicago, IL, USA), was used for statistical analyses.

\section{Results}

No differences in baseline clinical test characteristics, with the exception of platelet counts, were observed among patients who acquired complete renal recovery, partial renal recovery, or who remained dialysis dependent In our study, all patients were dialysis dependent on admission. After standardized treatment, 25 patients obtained dialysis independence within 3 months. Among the 25 patients who got dialysis independence, 13 patients obtained complete renal recovery $\left(\mathrm{CR}, \mathrm{eGFR}>=60 \mathrm{~mL} / \mathrm{min} / 1.73 \mathrm{~m}^{2}\right)$ and the other 12 patients acquired partial renal recovery 
(PR, $\left.15 \mathrm{~mL} / \mathrm{min} / 1.73 \mathrm{~m}^{2}<\mathrm{eGFR}<60 \mathrm{~mL} / \mathrm{min} / 1.73 \mathrm{~m}^{2}\right)$. As shown in Table 1, there were no differences of the clinical laboratory characteristics, with the exception of the platelet counts, among the 3 groups. Patients with renal recovery had higher platelet counts (CR: $326.3 \pm 160.410^{9} / \mathrm{L}$, PR: $\left.269.3 \pm 137.110^{9} / \mathrm{L}\right)$ than patients who remained dialysis dependent $\left(201.2 \pm 66.410^{9} / \mathrm{L}, P=0.040\right)$. There was a trend of higher hemoglobin in patients with renal recovery (CR: $8.5 \pm 1.7 \mathrm{~g} / \mathrm{L}, \mathrm{PR}: 7.9 \pm 1.0 \mathrm{~g} / \mathrm{L}$ ) compared to those who remained dialysis dependent $(7.3 \pm 1.5 \mathrm{~g} / \mathrm{L})$, but the difference was not statistically significant $(P=0.095)$.
Renal histopathological characteristics were strongly associated with the kidney prognosis

All patients received kidney biopsy, and all biopsy specimens contained more than 10 glomeruli. As shown in Table 2, the percentages of normal glomeruli and cellular crescent of patients with CR or PR were significantly higher than the patients who remained dialysis dependent $(P=0.007$ and $P=0.015)$, while the percentages of fibrous crescent and global glomerulosclerosis of patients with CR or PR were significantly lower than those of patients who remained dialysis dependent (both

Table 1 Comparison of baseline clinical laboratory characteristics of patients with renal recovery and patients remaining on dialysis within 3 months

\begin{tabular}{|c|c|c|c|c|}
\hline Feature & $\begin{array}{l}\text { Patients with complete renal recovery } \\
(n=13)\end{array}$ & $\begin{array}{l}\text { Patients with partial renal recovery } \\
(n=12)\end{array}$ & $\begin{array}{l}\text { Patients remaining on dialysis } \\
(n=15)\end{array}$ & $P$ value \\
\hline Male/female & $9 / 4$ & $6 / 6$ & $6 / 9$ & 0.297 \\
\hline Age (years) & $63.1 \pm 9.5$ & $57.7 \pm 7.3$ & $55.9 \pm 15.1$ & 0.252 \\
\hline $\begin{array}{l}\text { Time from first symptom } \\
\text { (days) }\end{array}$ & $70.7 \pm 75.9$ & $110.0 \pm 112.4$ & $92.1 \pm 46.1$ & 0.674 \\
\hline BVAS & $23.3 \pm 6.3$ & $19.9 \pm 5.0$ & $22.0 \pm 6.3$ & 0.368 \\
\hline Fever $(\mathrm{Y} / \mathrm{N})$ & $8 / 5$ & $8 / 4$ & $8 / 7$ & 0.774 \\
\hline Weight loss (Y/N) & $5 / 6$ & $4 / 8$ & $5 / 10$ & 0.781 \\
\hline Muscle/joint (Y/N) & $3 / 10$ & $3 / 9$ & $3 / 12$ & 0.952 \\
\hline Skin $(Y / N)$ & $1 / 12$ & $0 / 12$ & $3 / 12$ & 0.215 \\
\hline $\begin{array}{l}\text { Eyes/mucous membranes } \\
(\mathrm{Y} / \mathrm{N})\end{array}$ & $3 / 10$ & $2 / 10$ & $3 / 12$ & 0.923 \\
\hline Ear/nose/throat (Y/N) & $6 / 7$ & $5 / 7$ & $4 / 11$ & 0.534 \\
\hline Lung $(\mathrm{Y} / \mathrm{N})$ & $8 / 5$ & $4 / 8$ & $7 / 8$ & 0.368 \\
\hline Cardiovascular (Y/N) & $1 / 12$ & $0 / 12$ & $0 / 15$ & 0.345 \\
\hline Digestive tract $(\mathrm{Y} / \mathrm{N})$ & $2 / 11$ & $4 / 8$ & $2 / 13$ & 0.382 \\
\hline Nervous system $(\mathrm{Y} / \mathrm{N})$ & $3 / 10$ & $2 / 10$ & $2 / 13$ & 0.792 \\
\hline Gross hematuria (Y/N) & $0 / 13$ & $2 / 10$ & $1 / 14$ & 0.283 \\
\hline ANCA level (IU/mL) & $89.3 \pm 21.7$ & $101.7 \pm 36.0$ & $94.9 \pm 34.0$ & 0.847 \\
\hline $\mathrm{eGFR}\left(\mathrm{mL} / \mathrm{min} / 1.73 \mathrm{~m}^{2}\right)$ & $7.0 \pm 2.3$ & $6.6 \pm 2.1$ & $5.7 \pm 1.8$ & 0.231 \\
\hline Proteinuria (g/24 h) & $2.8 \pm 2.3$ & $2.5 \pm 3.0$ & $3.5 \pm 1.7$ & 0.641 \\
\hline Hemoglobumin (g/L) & $8.5 \pm 1.7$ & $7.9 \pm 1.0$ & $7.3 \pm 1.5$ & 0.095 \\
\hline White blood cell $\left(10^{9} / L\right)$ & $9.3 \pm 2.7$ & $8.0 \pm 3.1$ & $10.2 \pm 6.8$ & 0.507 \\
\hline Platelet $\left(10^{9} / \mathrm{L}\right)$ & $326.3 \pm 160.4$ & $269.3 \pm 137.1$ & $201.2 \pm 66.4$ & 0.040 \\
\hline $\mathrm{ESR}(\mathrm{mm} / \mathrm{h})$ & $83.2 \pm 41.0$ & $71.9 \pm 39.4$ & $82.5 \pm 45.2$ & 0.756 \\
\hline Serum albumin (g/L) & $28.4 \pm 5.5$ & $27.3 \pm 7.3$ & $28.1 \pm 6.9$ & 0.275 \\
\hline Serum calcium (mmol/L) & $2.0 \pm 0.4$ & $1.9 \pm 0.4$ & $1.8 \pm 0.4$ & 0.374 \\
\hline Serum phosphate (mmol/L) & $1.7 \pm 0.4$ & $1.7 \pm 0.4$ & $1.7 \pm 0.3$ & 0.215 \\
\hline Serum PTH (pg/mL) & $128.4 \pm 95.3$ & $131.4 \pm 86.3$ & $137.0 \pm 93.4$ & 0.114 \\
\hline Increased RF (Y/N) & $7 / 6$ & $3 / 9$ & $3 / 12$ & 0.130 \\
\hline C-reactive protein (mg/dL) & $4.6(0.9,15.6)$ & $1.8(0.2,8.7)$ & $1.9(0.3,16.3)$ & 0.105 \\
\hline Complement 3 (mg/dL) & $88.7 \pm 33.3$ & $82.7 \pm 23.9$ & $89.0 \pm 23.6$ & 0.896 \\
\hline Complement 4 (mg/dL) & $19.0 \pm 8.3$ & $23.8 \pm 3.2$ & $30.7 \pm 8.0$ & 0.113 \\
\hline
\end{tabular}

ANCA Antineutrophil cytoplasmic antibody, BVAS Birmingham vasculitis activity score, eGFR Estimated glomerular filtration rate, ESR Erythrocyte sedimentation rate, PTH Parathyroid hormone, RF Rheumatoid factor 
Table 2 Comparison of renal histopathological characteristics of patients with renal recovery and patients remaining on dialysis within 3 months

\begin{tabular}{|c|c|c|c|c|}
\hline Feature & $\begin{array}{l}\text { Patients with complete renal recovery } \\
(n=13)\end{array}$ & $\begin{array}{l}\text { Patients with partial renal recovery } \\
(n=12)\end{array}$ & $\begin{array}{l}\text { Patients remaining on dialysis } \\
(n=15)\end{array}$ & $\begin{array}{l}P \\
\text { value }\end{array}$ \\
\hline Normal glomeruli (\%) & $24.2(0.0,57.2)$ & $11.3(0.0,40.0)$ & $0.0(0.0,15.2)$ & 0.007 \\
\hline Fibrinoid necrosis (\%) & $3.8(0.0,16.7)$ & $0.0(0.0,10.0)$ & $0.0(0.0,3.1)$ & 0.065 \\
\hline Cellular crescent (\%) & $58.1(19.0,100.0)$ & $50.8(20.0,80.0)$ & $31.7(3.9,65.6)$ & 0.015 \\
\hline Fibrous crescent (\%) & $6.3(0.0,40.7)$ & $18.8(9.7,31.4)$ & $40.3(0.0,82.9)$ & $\begin{array}{l}< \\
0.001\end{array}$ \\
\hline $\begin{array}{l}\text { Global glomerulosclerosis } \\
(\%)\end{array}$ & $0.0(0.0,33.3)$ & $2.5(0.0,14.8)$ & $20.0(0.0,40.0)$ & $\begin{array}{l}< \\
0.001\end{array}$ \\
\hline $\begin{array}{l}\text { Interstitial infiltrates } \\
(-/+/++/+++)\end{array}$ & $1 / 2 / 6 / 4$ & $1 / 0 / 5 / 6$ & $0 / 2 / 8 / 5$ & 0.694 \\
\hline Interstitial fibrosis $(-/+/++)$ & $5 / 7 / 1$ & $2 / 5 / 5$ & $1 / 8 / 6$ & 0.133 \\
\hline Tubular atrophy $(-/+/++)$ & $1 / 7 / 5$ & $0 / 3 / 9$ & $0 / 4 / 11$ & 0.213 \\
\hline Arterial wall thickening $(-/+)$ & $5 / 8$ & $0 / 12$ & $0 / 15$ & 0.003 \\
\hline
\end{tabular}

$P<0.001)$. All patients with PR or patients who remained dialysis dependent had arterial wall thickening, while $38.5 \%$ of patients with CR did not have arterial wall thickening $(P=0.003)$.

\section{Mixed class of pathological classification is associated with poor kidney outcome}

The distributions of 4 pathological categories were investigated. No sclerotic class was found among the 40 patients. Only 2 patients were diagnosed as focal class, and both obtained renal recovery within 3 months. Among 18 patients in the crescentic class, 15 acquired renal recovery within 3 months and the other 3 stayed dialysis dependent. Among the 20 patients in the mixed class, 8 got renal recovery and the other 12 kept dialysis dependent. The renal recovery rate of the mixed class was significantly lower than that of crescentic class $(p=0.006)$ (Fig. 2a).
We compared the eGFR at admission, at 3 months, and at 1 year among patients in the different pathological categories. There was no difference in baseline eGFR among patients with focal, crescentic or mixed class. After 3 months of treatment, the eGFR of all 3 groups increased, but the eGFR of patients in the mixed class was lower than that of patients in the crescentic or focal classes. After following up for 1 year, 4 out of 18 patients in the crescentic class died, while 3 out of 20 patients in the mixed class died. Among the living patients, the eGFR of mixed class patients was still lower than that of the crescentic and focal classes (Fig. 2b).

Since 12 patients who recovered renal function obtained PR but not CR, the renal survival of these patients at 1 year was analyzed. We observed that 3 patients with mixed class entered ESRD, and these 3 patients had higher a proportion of global glomerulosclerosis than patients who did not enter ESRD (Table 3).
A

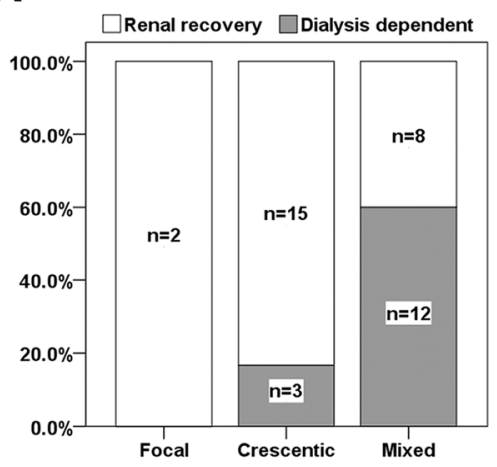

B

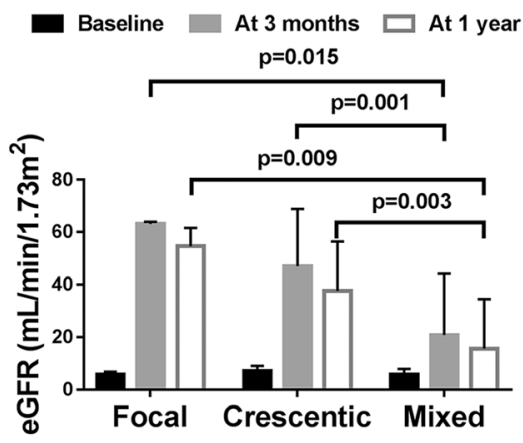

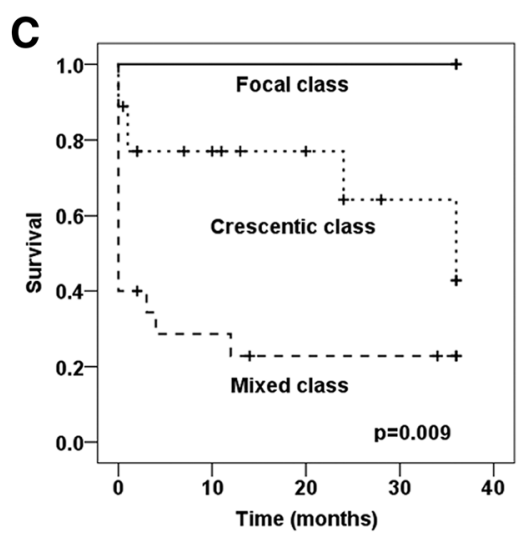

C

Fig. 2 Kidney prognosis of patients with different histopathologic classification. a The renal recovery rate of patients with focal, crescentic or mixed class. b The estimated glomerular filtration rate (eGFR) at admission, 3 months after therapy and 1 year after therapy of patients with focal, crescentic or mixed class. c Comparison of the combined end point (Death and ESRD) among patients with different histopathologic classification. The longest follow-up time was 36 months 
Table 3 Comparison of the baseline renal histopathological characteristics between patients who entered ESRD and who did not enter ESRD after follow-up for 1 year among 12 patients with partial renal recovery

\begin{tabular}{llll}
\hline Feature & Patients who entered ESRD $(n=3)$ & Patients who did not enter ESRD $(n=9)$ & $P$ value \\
\hline Normal glomeruli (\%) & $0.0(0.0,4.0)$ & $14.3(5.0,36.7)$ & 0.405 \\
Fibrinoid necrosis (\%) & $0.0(0.0,0.0)$ & $0.0(0.0,10.0)$ & 0.275 \\
Cellular crescent (\%) & $48.6(20.0,80.0)$ & $51.5(33.3,74.2)$ & 0.782 \\
Fibrous crescent (\%) & $30.0(13.3,31.4)$ & $18.2(9.7,30.0)$ & 0.195 \\
Global glomerulosclerosis (\%) & $0.0(0.0,14.8)$ & $10.0(6.7,14.3)$ & 0.038 \\
Pathological classification (F/C/M/S) & $0 / 0 / 3 / 0$ & $0 / 6 / 3 / 0$ & 0.046 \\
\hline
\end{tabular}

F/C/M/S Focal/crescentic/mixed/sclerotic

We then compared the combined end point (Death and ESRD) among patients with different pathological categories. As shown in Fig. 2c, the order of the survival was focal, crescentic and mixed classes $(p=0.009)$.

\section{Percentage of fibrous crescent+global glomerulosclerosis} was a marker to predict the kidney outcome

ROC curves were calculated to explore whether there were pathological parameters which could predict the renal prognosis. The ROC curves are shown in Fig. 3a. The AUC of global glomerulosclerosis was the greatest (0.853) among all 4 glomerular parameters. Since fibrous crescent usually indicates a kind of irreversible lesion, we attempted to determine whether the combination of fibrous crescent and global glomerulosclerosis could increase the accuracy of the diagnosis. As shown in Fig. 3b, the AUC of fibrous crescent+global glomerulosclerosis was greater than any other parameter. When the percentage of fibrous crescent+global glomerulosclerosis was $32.6 \%$, the Youden index was at the peak value (sensitivity was $93.3 \%$ and specificity was $88.0 \%$ ). When the percentage of fibrous crescent+global glomerulosclerosis exceeded $47.9 \%$, renal recovery was unlikely.

Increased peripheral platelet counts were associated with reversible pathological lesions and high renal recovery rate.

Since patients who obtained renal recovery had higher platelet counts than patients who were dialysis dependent, we explored whether platelet counts could be used as a substitute for pathological lesions to determine kidney outcome. Correlation analysis indicated that platelet counts negatively correlated with the percentage of fibrous crescent+global glomerulosclerosis (Fig. 4a). There were no differences in the distributions of focal, crescentic and

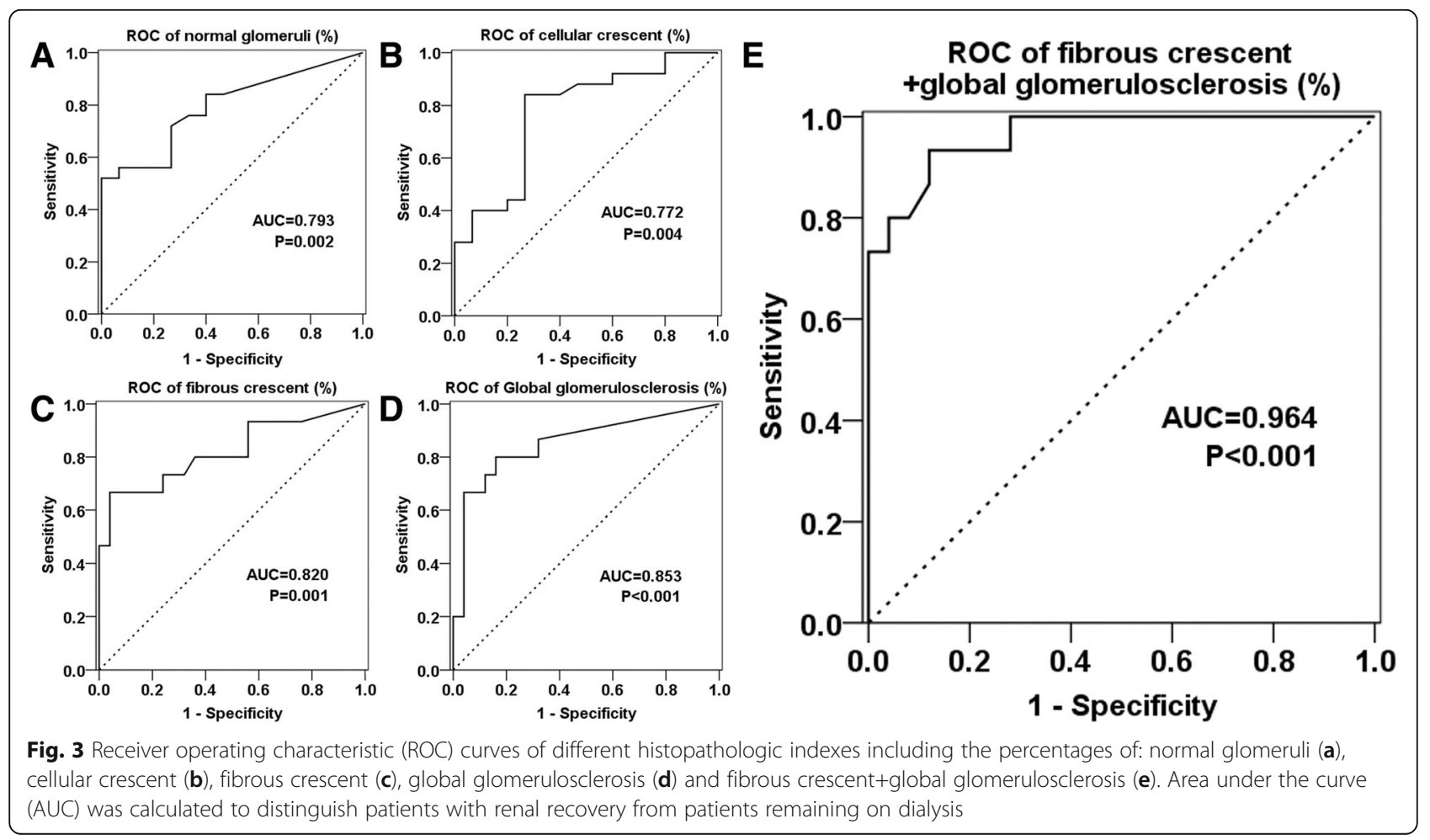


A

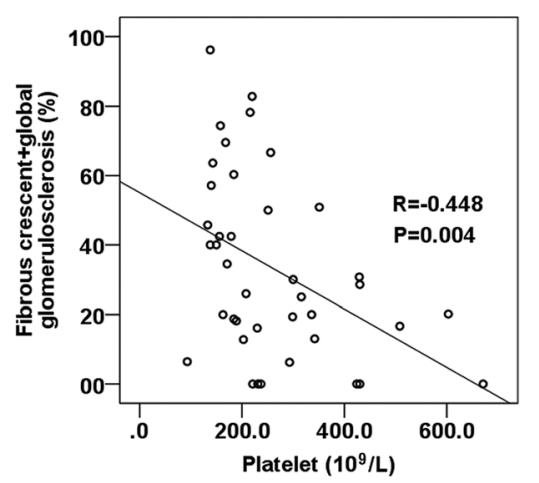

C

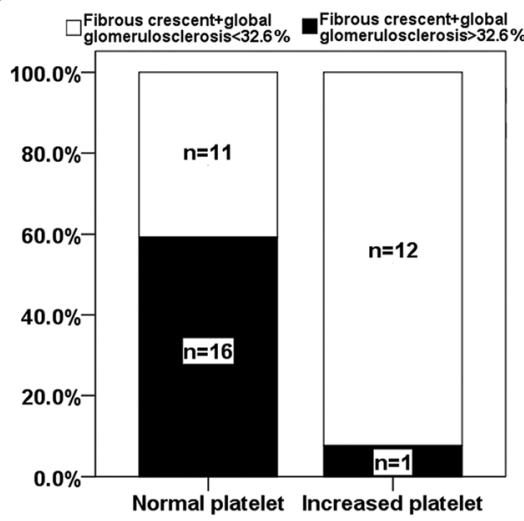

B

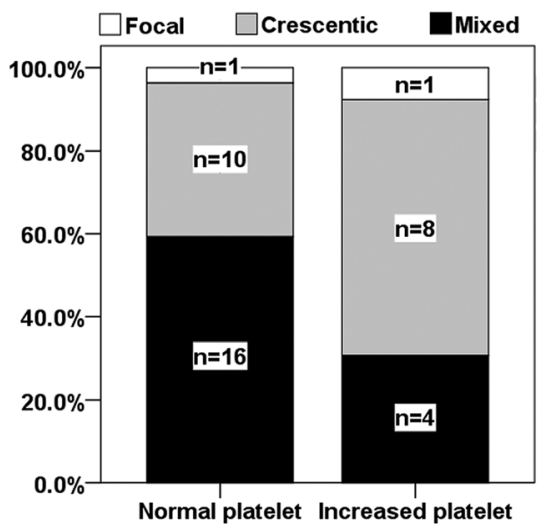

D

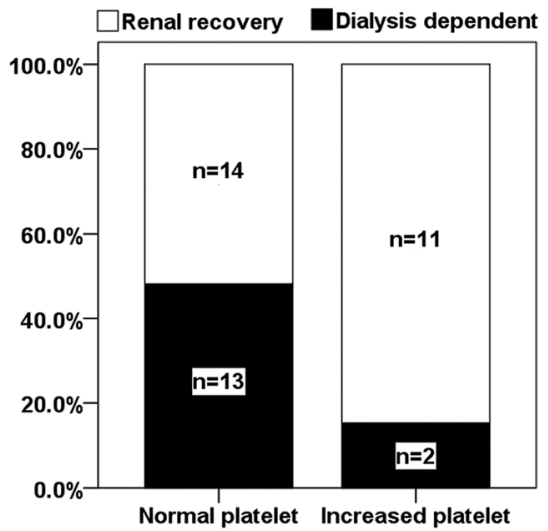

Fig. 4 Relationship between platelet counts and histopathologic indexes and relationship between platelet counts and prognosis. a Correlation between platelet counts and the percentages of fibrous crescent+global glomerulosclerosis. $\mathbf{b}$ Comparison of the difference of histopathologic classification between patients with normal and increased platelets. Increased platelets were diagnosed when platelets were more than the upper limit $300 \times 10^{9} / \mathrm{L}$. c Comparison of the proportions of patients with fibrous crescent+global glomerulosclerosis more than cut-off value ( $32.6 \%$ ) between patients with normal and increased platelets. $\mathbf{d}$ Comparison of the proportions of renal recovery within 3 months between patients with normal and increased platelets

mixed categories between patients with normal platelets and patients with increased platelets (Increased platelets meant the platelet count was larger than the upper limit of normal range $300 \times 10^{9} / \mathrm{L}$ ) (Fig. $4 \mathrm{~b}, p=0.238$ ), but for the majority patients with increased platelets, the percentages of fibrous crescent+global glomerulosclerosis were less than $32.6 \%$ (Fig. 4c, $p=0.002$, compared with patients with normal platelets). Correspondingly, most patients with increased platelets obtained renal recovery (CR + PR) within 3 months (Fig. $4 \mathrm{~d}, p=0.045$, compared with patients with normal platelets). However, the AUC of platelet counts for distinguishing renal recovery from dialysis dependence was only 0.709 .

\section{PE treatment accelerated the renal recovery}

Effects of different therapy regimens on renal recovery were analyzed. All 40 patients received daily oral corticosteroid as a primary treatment. Among these patients, 7 received MP + pulse CTX treatment, 19 patients received $\mathrm{MP}+$ oral CTX treatment, 3 patients received $\mathrm{PE}+$ pulse CTX treatment and 11 patients received PE + oral CTX treatment. As shown in Table 4, there were no significant differences in the baseline BVAS, eGFR, proteinuria, or incidence of gross hematuria between the $\mathrm{MP}$ and $\mathrm{PE}$ groups or between pulse CTX and oral CTX groups. There were also no significant differences in kidney histopathology parameters among groups (data not shown). Compared with MP, PE accelerated the rate of renal recovery $(29.4 \pm 15.6$ vs. $41.4 \pm 11.7$ days, $p=0.039)$, and there was a tendency for a greater renal recovery rate in PE group (79\%) compared with MP group (53\%).

\section{Discussion}

RPGN is the clinical manifestation of several types of kidney diseases, including anti-glomerular basement membrane antibody disease (type 1 RPGN), AAV (type 2 
Table 4 Influences of different therapy regimens on the renal recovery within 3 months

\begin{tabular}{|c|c|c|c|c|c|c|}
\hline & \multicolumn{3}{|l|}{ MP vs. PE } & \multicolumn{3}{|c|}{ Pulse CTX vs. Oral CTX } \\
\hline & $\mathrm{MP}(n=26)$ & $\operatorname{PE}(n=14)$ & $P$ value & Pulse CTX $(n=10)$ & Oral CTX $(n=30)$ & $P$ value \\
\hline $\mathrm{MP} / \mathrm{PE}$ & - & - & - & $7 / 3$ & 19/11 & 0.702 \\
\hline Pulse CTX/Oral CTX & $7 / 19$ & $3 / 11$ & 0.702 & - & - & - \\
\hline BVAS & $22.9 \pm 6.2$ & $19.9 \pm 5.1$ & 0.132 & $23.4 \pm 4.9$ & $21.3 \pm 6.2$ & 0.333 \\
\hline eGFR (mL/min/1.73 m2) & $6.4 \pm 2.0$ & $6.2 \pm 2.3$ & 0.774 & $6.6 \pm 2.1$ & $6.3 \pm 2.1$ & 0.646 \\
\hline Proteinuria (g/24 h) & $2.7 \pm 1.7$ & $3.3 \pm 3.2$ & 0.384 & $2.8 \pm 2.1$ & $3.0 \pm 2.5$ & 0.848 \\
\hline Gross hematuria $(\mathrm{Y} / \mathrm{N})$ & $3 / 23$ & $0 / 14$ & 0.186 & $1 / 9$ & $2 / 28$ & 0.729 \\
\hline Renal recovery (CR/PR/N) & $7 / 7 / 12$ & $6 / 5 / 3$ & 0.297 & $3 / 4 / 3$ & 10/8/12 & 0.715 \\
\hline Time for getting dialysis independence (days) & $41.4 \pm 11.7$ & $29.4 \pm 15.6$ & 0.039 & $34.8 \pm 11.1$ & $36.3 \pm 15.9$ & 0.894 \\
\hline
\end{tabular}

BVAS Birmingham vasculitis activity score, CTX Cyclophosphamide, CR Complete remission, eGFR Estimated glomerular filtration rate, MP Methylprednisolone pulse therapy, $P E$ Plasma exchange, $P R$ Partial remission

RPGN), and RPGN with glomerular deposition of immune complexes (type 3 RPGN) [13]. Patients requiring dialysis at disease onset caused by type 1 RPGN usually rapidly enter ESRD [14, 15]. In contrast with type 1 RPGN, most patients with type 3 RPGN usually develop ESRD gradually. As for type 2 RPGN (ANCA-GN), the kidney outcome varies for different individuals.

In the current study, we found that pathological severity was the most important factor affecting renal outcome. Currently, the only practical tool for estimating the renal prognosis of ANCA-GN is the histopathologic classification proposed in 2010 [8]. Its clinical value has been evaluated by several studies in recent years [16-29]. Interestingly, the distribution of the four renal histological categories in our study is different from all previous studies. In the previous studies, the 4 pathological categories were approximately evenly spread, while in our cohort the crescent and mixed classes accounted for a near majority of patients. Although the initial study of Berden AE et al. suggested the prognosis of the mixed class was worse than that of the crescentic class [8], there is no consistent conclusion in subsequent validation studies concerning the comparison of the prognosis between the mixed and crescentic classes. In our study, the renal outcome of the mixed class was significantly worse than that of the crescentic class.

According to previous studies, the sclerotic class (glomerulosclerosis over 50\%) had the worst prognosis in ANCA-GN, but most patients took several years to develop ESRD. However, the patients in our study developed ESRD directly when fibrous crescent+glomerulosclerosis was greater than $32.6 \%$. These results were very interesting and required interpretation. Theoretically, not all glomeruli with cellular crescents could return to normal even following intensive immunosuppressive treatments. There were only two patients in the focal class in this study, and we speculate that the proportions of normal glomeruli in both the mixed and crescentic classes in our study were much lower than the patients of the mixed and crescentic classes in previous studies. In contrast, since the proportion of chronic lesions was greater in the mixed class than in the crescentic class, it was not difficult to see why the renal prognosis of the mixed class was inferior to that of the crescentic class in our study. Therefore, for patients needing dialysis at disease onset, the renal outcomes of the crescentic and mixed classes are significantly different.

In clinical work, not all patients are able to undergo kidney biopsy, so an alternative biomarker is of great importance [30, 31]. Platelet count has not been a significant factor in previous studies, but these studies included both patients needing dialysis and patients who did not need dialysis. In our study, platelet count was the only laboratory parameter which exhibited a significant difference between patients who obtained renal recovery and those who remained dialysis dependent. It is noteworthy that platelet counts have been associated with disease activity in AAV and can distinguish acute infection from active disease [32, 33]. Platelet-leukocyte aggregates were significantly higher thrombocytosis. Most importantly, platelets could activate the alternative complement pathway, which is crucial in the pathogenesis of AAV [34]. The peripheral levels of platelet-derived microparticles (PMPs) were significantly associated with disease activity and the proportion of crescents in the renal specimen [35]. We found platelet counts correlated with the percentages of fibrous crescent+global glomerulosclerosis. However, there was not a suitable cut-off value for platelet counts to predict kidney outcome. In addition, the diagnosis of thrombocytosis was influenced by the definition of upper normal limit of platelets. In our hospital, thrombocytosis was diagnosed when platelet counts were greater than $300 \times 10^{9} / \mathrm{L}$. Thus, platelet counts cannot replace pathological parameters to predict renal prognosis in AAV.

CRP is a classical acute-phase protein. Interestingly, although there was a correlation between platelets and CRP (data not shown), there was no significant difference in CRP among patients with different renal outcomes. One 
possible explanation is that CRP is more easily affected by other factors (such as potential infection) than platelets [32].

In the treatment of ANCA-GN, PE has been recommended due to its superiority to MP [7]. In our study, we also demonstrated that patients receiving PE had a faster renal recovery than patients receiving MP, but a higher remission rate was not observed. This may be due to the small cohort size of our study. However, the largest trial of AAV to date (PEXIVAS study), which included 704 participants from 98 sites in 15 countries, indicated no improvement in outcomes (death or ESRD) of patients who received PE. This issue requires further investigation.

Several limitations of our study should be mentioned. Firstly, due to the restrictions of the enrollment standard, the sample size of the study was small. In our study, no sclerotic class patients were identified, but as many as twenty-eight patients who were dialysis dependent at disease onset were excluded because they did not receive kidney biopsy. Among these 28 patients, 12 patients had kidney atrophy and did not recover kidney function within 3 months. We could not exclude the possibility that most, or even all of these patients were of the sclerotic class. Secondly, only MPO-ANCA positive patients were included because PR3-ANCA positive AAV is relatively rare in our center. So whether the present results pertain to patients with PR3-ANCA positive AAV is unclear. Thirdly, being limited by the inclusion standard, all patients without kidney biopsy or patients that died within 3 months were excluded. This affected the estimation of the effects of different therapies on renal recovery. Finally, whether patients received either MP or $\mathrm{PE}$ treatment was not randomly determined due to the shortage of plasma.

\section{Conclusions}

In conclusion, for ANCA-GN requiring dialysis at disease onset, the crescentic and mixed classes account for the majority of patients in our cohort. Mixed class is associated with worse prognosis than crescentic class. A high level of fibrous crescent+global glomerulosclerosis pathology is a predictor of dialysis dependence. Patients with increased platelet counts have a greater number of acute pathological lesions and higher renal recovery rate compared to patients with normal platelet counts.

\footnotetext{
Abbreviations

AAV: Anti-neutrophil cytoplasmic antibody-associated vasculitis; ANCA: Antineutrophil cytoplasmic antibody; AUC: Area under the curve; BVAS: Birmingham Vasculitis Activity Score; CR: Complete renal recovery; CTX: Cyclophosphamide; eGFR: Estimated glomerular filtration rate; ESRD: End stage renal disease; GN: Glomerulonephritis; MP: Methylprednisolone pulse therapy; MPO: Myeloperoxidase; PE: Plasma exchange; PMPs: platelet-derived microparticles; PR: Partial renal recovery; PR3: Proteinase 3; ROC: Receiver operating characteristics; RPGN: Rapidly progressive glomerulonephritis
}

\section{Acknowledgements}

The authors thank Wen-ya Shang (Department of Nephrology; Tianjin Medical University General Hospital) for providing the data of the kidney biopsy.

\section{Authors' contributions}

PCX conceived and designed the experiments, analyzed data, and wrote the paper. TC collected data, analyzed data, and wrote the paper. SJW, XY and SG made substantial contributions to the acquisition of data, and the analysis and interpretation of data. SYH performed the statistical analyses. LW performed the diagnosis of kidney biopsy. TKY assisted the diagnosis of pathology, raised lots of important and helpful suggestions in manuscript writing. All authors read and approved the final manuscript.

\section{Funding}

This study is supported by three grants of National Natural Science Fund (No. 81200534, No. 81570630 and No. 81600554), two grants of Tianjin Research Program of Application Foundation and Advanced Technology (No. 15JCQNJC10700 and 17JCQNJC10200) and a grant of China Postdoctoral Science Foundation funded project (No. 2014 M560191). The funders have no role in study design, data collection and analysis, decision to publish, or preparation of the manuscript.

\section{Availability of data and materials}

The datasets used and/or analyzed during the current study are available from the corresponding author on reasonable request.

\section{Ethics approval and consent to participate}

The research was in compliance of the declaration of Helsinki and the protocol was approved by the institutional review board of Tianjin Medical University General Hospital [ZYY-IRB-SOP-016(F)-002-03]. Informed consent from each patient to participate in this study was not required by the Institutional Review Board, because the study was a retrospective review of clinical records only.

\section{Consent for publication}

Not applicable because this report does not contain data for individual patients.

\section{Competing interests}

The authors declare that they have no competing interests.

\section{Author details}

'Department of Nephrology, Tianjin Medical University General Hospital, Tianjin 300052, China. ${ }^{2}$ Department of Hematology, Tianjin Medical University General Hospital, Tianjin 300052, China.

Received: 17 September 2018 Accepted: 24 July 2019

Published online: 30 July 2019

\section{References}

1. Tang W, Bose B, McDonald SP, et al. The outcomes of patients with ESRD and ANCA-associated vasculitis in Australia and New Zealand. Clin J Am Soc Nephrol. 2013;8:773-80.

2. Romeu M, Couchoud C, Delaroziere JC, et al. Survival of patients with ANCA-associated vasculitis on chronic dialysis: data from the French REIN registry from 2002 to 2011. QJM. 2014;107:545-55.

3. Kallenberg CG. Key advances in the clinical approach to ANCA-associated vasculitis. Nat Rev Rheumatol. 2014;10:484-93.

4. Sinico RA, Di Toma $L$, Radice A. Renal involvement in anti-neutrophil cytoplasmic autoantibody associated vasculitis. Autoimmun Rev. 2013;12: 477-82.

5. Booth AD, Almond MK, Burns A, et al. Outcome of ANCA-associated renal vasculitis: a 5-year retrospective study. Am J Kidney Dis. 2003;41:776-84.

6. Shi YY, Li ZY, Zhao MH, et al. The CD4 lymphocyte count is a better predictor of overall infection than the Total lymphocyte count in ANCAassociated Vasculitis under a corticosteroid and cyclophosphamide regimen: a retrospective cohort. Medicine (Baltimore). 2015;94:e843.

7. Jayne DR, Gaskin G, Rasmussen N, et al. Randomized trial of plasma exchange or high-dosage methylprednisolone as adjunctive therapy for severe renal vasculitis. J Am Soc Nephrol. 2007;18:2180-8. 
8. Berden AE, Ferrario F, Hagen EC, et al. Histopathologic classification of ANCA-associated glomerulonephritis. J Am Soc Nephrol. 2010;21:1628-36.

9. Jennette JC, Falk RJ, Bacon PA, et al. 2012 revised international Chapel Hill consensus conference nomenclature of Vasculitides. Arthritis Rheum. 2013; 65:1-11.

10. Xu PC, Chen T, Gao S, et al. Clinical and pathologic characteristics of pauciimmune anti-myeloperoxidase antibody associated glomerulonephritis with nephrotic range proteinuria. Ren Fail. 2018;40:554-60.

11. Li ZY, Gou SJ, Chen M, et al. Predictors for outcomes in patients with severe ANCA-associated glomerulonephritis who were dialysis-dependent at presentation: a study of 89 cases in a single Chinese center. Semin Arthritis Rheum. 2013;42:515-21.

12. Ma TT, Liu YR, Chen $M$, et al. Late restoration of renal function in patients with severe ANCA-associated glomerulonephritis who were dialysisdependent at presentation. Clin Rheumatol. 2018:37:2143-50.

13. Gharbi C, Bourry E, Rouvier $P$, et al. Rapidly progressive lupus nephritis and concomitant thrombotic microangiopathy. Clin Exp Nephrol. 2010; 14:487-91.

14. Zhao J, Yang R, Cui Z, et al. Characteristics and outcome of Chinese patients with both antineutrophil cytoplasmic antibody and antiglomerular basement membrane antibodies. Nephron Clin Pract. 2007;107:c56-62.

15. Cui Z, Zhao MH, Xin G, et al. Characteristics and prognosis of Chinese patients with anti-glomerular basement membrane disease. Nephron Clin Pract. 2005;99:C49-55.

16. Chang DY, Wu LH, Liu G, et al. Re-evaluation of the histopathologic classification of ANCA-associated glomerulonephritis: a study of 121 patients in a single center. Nephrol Dial Transplant. 2012;27:2343-9.

17. Córdova-Sánchez BM, Mejía-Vilet JM, Morales-Buenrostro LE, et al. Clinical presentation and outcome prediction of clinical, serological, and histopathological classification schemes in ANCA-associated vasculitis with renal involvement. Clin Rheumatol. 2016;35:1805-16.

18. Ellis $C L$, Manno RL, Havill JP, et al. Validation of the new classification of pauci-immune glomerulonephritis in a United States cohort and its correlation with renal outcome. BMC Nephrol. 2013;14:210.

19. Hilhorst M, Wilde B, van Breda Vriesman $P$, et al. Estimating renal survival using the ANCA-associated GN classification. J Am Soc Nephrol. 2013;24: 1371-5.

20. Iwakiri T, Fujimoto S, Kitagawa $\mathrm{K}$, et al. Validation of a newly proposed histopathological classification in Japanese patients with anti-neutrophil cytoplasmic antibody-associated glomerulonephritis. BMC Nephrol. 2013;14:125.

21. Kristensen T, Gregersen JW, Krag SR, et al. The relation between histopathological classification and renal outcome, ANCA subtype and treatment regimens in ANCA-associated vasculitis. Clin Exp Rheumatol. 2016;34:S105-10.

22. Moroni G, Binda V, Leoni A, et al. Predictors of renal survival in ANCAassociated vasculitis. Validation of a histopatological classification schema and review of the literature. Clin Exp Rheumatol. 2015;33:S-56-63.

23. Muso E, Endo T, Itabashi M, et al. Evaluation of the newly proposed simplified histological classification in Japanese cohorts of myeloperoxidaseanti-neutrophil cytoplasmic antibody-associated glomerulonephritis in comparison with other Asian and European cohorts. Clin Exp Nephrol. 2013; 17:659-62.

24. Naidu GS, Sharma A, Nada R, et al. Histopathological classification of pauciimmune glomerulonephritis and its impact on outcome. Rheumatol Int. 2014:34:1721-7.

25. Nohr E, Girard L, James M, et al. Validation of a histopathologic classification scheme for antineutrophil cytoplasmic antibody-associated glomerulonephritis. Hum Pathol. 2014;45:1423-9.

26. Quintana LF, Peréz NS, De Sousa E, et al. ANCA serotype and histopathological classification for the prediction of renal outcome in ANCA-associated glomerulonephritis. Nephrol Dial Transplant. 2014;29:1764-9.

27. Tanna A, Guarino L, Tam FW, et al. Long-term outcome of anti-neutrophil cytoplasm antibody-associated glomerulonephritis: evaluation of the international histological classification and other prognostic factors. Nephrol Dial Transplant. 2015;30:1185-92.

28. Togashi M, Komatsuda A, Nara M, et al. Validation of the 2010 histopathological classification of ANCA-associated glomerulonephritis in a Japanese single-center cohort. Mod Rheumatol. 2014;24:300-3.

29. Bjørneklett R, Sriskandarajah S, Bostad L. Prognostic value of histologic classification of ANCA-associated glomerulonephritis. Clin J Am Soc Nephrol. 2016;11:2159-67.
30. Westman KW, Bygren PG, Olsson H, et al. Relapse rate, renal survival, and cancer morbidity in patients with Wegener's granulomatosis or microscopic polyangiitis with renal involvement. J Am Soc Nephrol. 1998;9:842-52.

31. Chen Y, Bao H, Liu Z, et al. Risk factors for renal survival in Chinese patients with myeloperoxidase-ANCA-associated GN. Clin J Am Soc Nephrol. 2017;12: 417-25.

32. Willeke $P$, Kumpers $P$, Schluter $B$, et al. Platelet counts as a biomarker in ANCA-associated vasculitis. Scand J Rheumatol. 2015;44:302-8.

33. Miao D, Li DY, Chen M, et al. Platelets are activated in ANCA-associated vasculitis via thrombin-PARs pathway and can activate the alternative complement pathway. Arthritis Res Ther. 2017;19:252.

34. Chen SF, Wang FM, Li ZY, et al. Complement factor $\mathrm{H}$ inhibits antineutrophil cytoplasmic autoantibody-induced neutrophil activation by interacting with neutrophils. Front Immunol. 2018;9:559.

35. Miao $\mathrm{D}, \mathrm{Ma} \mathrm{TT}$, Chen $\mathrm{M}$, et al. Platelets release proinflammatory microparticles in anti-neutrophil cytoplasmic antibody-associated vasculitis. Rheumatology (Oxford). 2019; Epub ahead of print.

\section{Publisher's Note}

Springer Nature remains neutral with regard to jurisdictional claims in published maps and institutional affiliations.

\section{Ready to submit your research? Choose BMC and benefit from:}

- fast, convenient online submission

- thorough peer review by experienced researchers in your field

- rapid publication on acceptance

- support for research data, including large and complex data types

- gold Open Access which fosters wider collaboration and increased citations

- maximum visibility for your research: over $100 \mathrm{M}$ website views per year

At BMC, research is always in progress.

Learn more biomedcentral.com/submissions 